\title{
THE CARDINALITY OF THE SET OF LEFT INVARIANT MEANS ON TOPOLOGICAL SEMIGROUPS
}

\author{
Heneri A.M. DzinotyiWeyi
}

\begin{abstract}
For a very large class of topological semigroups, we establish lower and upper bounds for the cardinality of the set of left invariant means on the space of left uniformly continuous functions. In certain cases we show that such a cardinality is exactly $2^{2^{b}}$, where $b$ is the smallest cardinality of the covering of the underlying topological semigroup by compact sets.
\end{abstract}

\section{INTRODUCTION}

Let $G$ be a non-compact locally compact topological group such that $L^{\infty}(G)$ admits a topologically left invariant mean, and let $T L I M(G)$ be the set of all such means. Various studies have looked at the size of such a set. In particular when $G$ is $\sigma$-compact, Chou [1] showed that $\operatorname{card}(T L I M(G)) \geqslant 2^{c}$, and when $G$ is discrete, he [2] showed that $\operatorname{card}(T L I M(G))=2^{2^{|G|}}$. (Here $c$ is the cardinality of the continuum and $|G|$ is the cardinality of $G$.) Generalising the latter result, Lau and Paterson [10] proved that $\operatorname{card}(T L I M(G))=2^{2^{b(G)}}$, where $b(G)$ is the smallest cardinality of a covering of $G$ by compact sets. (The idea of employing $b(G)$ has been used earlier by Liu and van Rooij [11].) Also for certain discrete semigroups, $S$, Klawe [9] showed that one may have the cardinality of the set of left invariant means on the bounded functions equal to $2^{2^{|S|}}$.

Now let $S$ be a topological semigroup, $L U C(S)$ the space of left uniformly continuous functions on $S$ and $M(S)$ the space of bounded Radon measures on $S$. Let $b(S)$ (or $d(S)$ ) be the cardinality of a covering of $S$ by compact (or respectively, relatively neo-compact) sets. (For definitions see section 2.) In this paper, for a large class of $S$, for which groups $G$ (as mentioned above) are a very special case, we show that the cardinality of the set of left invariant means on $L U C(S)$, and that of topologically left invariant means on $M(S)^{\star}$, lies between $2^{2^{b(S)}}$ and $2^{2^{d(S)}}$. For many cases we also have $b(S)=d(S)$.

Received 2 June 1987

Work in this paper was done during a Research visit at TH-Darmstadt. Thanks are due to the Alexander von Humboldt Foundation, for financial support, and to Professors K.H. Hofmann and K. Keimel, for inspiration.

Copyright Clearance Centre, Inc. Serial-fee code: 0004-9729/88 \$A2.00+0.00. 
In section 2, we collect together some definitions and preliminary results, and then formally state our results in section 3 . Some results employed at critical points of our proofs are collected into lemmas in section 4. Sections 5 to 7 contain proofs of the results stated in section 3 . We give a more detailed proof in section 5 upon which later proofs are moulded. We conclude the paper with a few remarks in section 8 .

For other results on sizes of sets of invariant means, the interested reader is referred to $[\mathbf{1}],[\mathbf{2}],[\mathbf{5}],[\boldsymbol{\theta}],[\mathbf{1 0}],[\mathbf{1 2}]$ and to the references mentioned therein.

\section{Preliminaries}

2.1 Terminology. By topological semigroup, we mean a semigroup $S$ endowed with a Hausdorff topology with respect to which the semigroup operation $(x, y) \rightarrow x y$ defines a jointly continuous mapping of $S \times S$ into $S$.

Let $S$ be a topological semigroup throughout this section.

For any subsets $A, B$ and $\{x\}$ of $S$, we employ the following notation:

$$
\begin{aligned}
A^{-1} B & :=\{y \in S: a y \in B \text { for some } a \in A\} \\
x^{-1} B & :=\{x\}^{-1} B \\
A^{-1} x & :=A^{-1}\{x\}
\end{aligned}
$$

By symmetry, one simularly defines right handed versions $B A^{-1}, B x^{-1}$ and $x A^{-1}$.

In particular, if $A_{1}, A_{2}, \ldots, A_{n}$ are subsets of $S$, we write

$$
\begin{aligned}
A_{1} \oplus A_{2} & :=\left\{A_{1} A_{2}, A_{1}^{-1} A_{2}, A_{1} A_{2}^{-1}\right\} \\
A_{1} \oplus A_{2} \oplus A_{3} & :=\left[\cup\left\{A_{1} \oplus B: B \in A_{2} \oplus A_{3}\right\}\right] \cup\left[\cup\left\{B \oplus A_{3}: B \in A_{1} \oplus A_{2}\right\}\right]
\end{aligned}
$$

and hence inductively define $A_{1} \oplus A_{2} \oplus \ldots \oplus A_{n}$.

Following [5] and [6], a subset $B$ of $S$ is said to be relatively neo-compact if $B$ is contained in a (finite) union of sets in $A_{1} \oplus A_{2} \oplus \cdots \oplus A_{n}$, for some compact sets $A_{1}, A_{2}, \ldots, A_{n}$ of $S$. In particular if $C^{-1} D$ and $D C^{-1}$ are compact sets, for all compact subsets $C$ and $D$ of $S$, then relatively neo-compact subsets are precisely the relatively compact subsets. For brevity, we write $\bar{B}$ for the closure of a set $B$ and $|B|$ for its cardinality.

Recall that $b(S)$ (respectively, $d(S)$ ) is the smallest possible cardinality of a covering of $S$ by compact (respectively, relatively neo-compact) subsets of $S$. We thus have $d(S) \leqslant b(S)$ always. Also the following result is evident; we quote it for easy reference.

2.2 Proposition. Let $S$ be non-relatively neo-compact. Then

(a) if $S$ is $\sigma$-compact, we have that $b(S)=d(S)=\aleph_{0}$.

(b) if the sets $C D^{-1}$ and $D C^{-1}$ are compact for all compact subsets $C$ and $D$ of $S$, then $b(S)=d(S)$. 
2.3 Notation. Let $C(S)$ denote the space of all bounded continuous complex-valued functions on $S$, and $M(S)$ the set of all bounded complex-valued regular Borel measures on $S$.

For every function $f$ in $C(S)$ and point $x$ in $S$, we have the functions ${ }_{x} f$ and $f_{x}$ in $C(S)$ given by

$$
{ }_{x} f(y):=f(x y) \text { and } f_{x}(y):=f(y x)(y \in S) .
$$

The space of left uniformly continuous functions on $S$ is

$L U C(S):=\left\{f \in C(S):\right.$ the map $x \rightarrow{ }_{x} f$ of $S$ into $C(S)$ is norm-continuous $\}$

Following [4] we say that $S$ is $C$-distinguished if the real-valued functions in $C(S)$ separate points.

For a $C$-distinguished $S$ we have $M(S)$ a Banach algebra under the usual total variation norm $\|\cdot\|$ and with convolution multiplication given by

$$
\nu^{\star} \mu(A):=\iint \chi_{A}(x y) d \nu(x) d \mu(y)
$$

for all $\nu, \mu \in M(S)$ and Borel subsets $A$ of $S$; where $\chi_{A}$ denotes the characteristic function of the set $A$. For $h$ in $M(S)^{\star}$ and $\nu, \mu$ in $M(S)$, we may then define $\nu o h$, $h o \mu$ and $\nu$ oho $\mu$ in $M(S)$ by

$$
\begin{aligned}
\nu o h(\eta) & :=h\left(\nu^{\star} \eta\right), \quad h o \mu(\eta):=\grave{h\left(\eta^{\star} \mu\right)} \\
\text { and } \nu o h o \mu(\eta) & :=h\left(\nu^{\star} \eta^{\star} \mu\right) \text { for all } \eta \in M(S) .
\end{aligned}
$$

In particular for $x \in S$ and $f \in C(S)$ we have that

$$
\delta_{x} \circ f={ }_{x} f,
$$

where $\delta_{x}$ denotes the point mass of $x$ and $f$ is identified with the functional in $M(S)^{\star}$ given by $\nu \rightarrow \int f(y) d \nu(y)$, for all $\nu \in M(S)$.

We define $\operatorname{supp}(\mu):=\{x \in S:|\mu|(V)>0$ for every open neighbourhood $V$ of $x\}$, for all $\mu$ in $M(S)$. In particular, for every subset $M_{0}$ of $M(S)$, by the foundation of $M_{0}$, we mean the closure of the $\operatorname{set} U\left\{\operatorname{supp}(\mu): \mu \in M_{0}\right\}$.

For any $S$, we define the set of absolutely continuous measures, $M_{a}(S)$, by

$$
M_{a}(S):=\left\{\mu \in M(S): \text { the maps } x \rightarrow|\mu|\left(x^{-1} K\right) \text { and } x \rightarrow|\mu|\left(K x^{-1}\right) \text { of } S\right.
$$$$
\text { into } R \text { are continuous, for all compact } K \subset S\} \text {. }
$$

(Here $|\mu|$ denotes the measure arising from the total variation of $\mu$.)

For semigroups $S, M_{a}(S)$ plays a role analogous to that played by the group algebra $L^{1}(G)$ for locally compact groups $G$. A comprehensive study of $M_{a}(S)$ can be found in [5].

The following definition is of particular importance in our paper: 
2.4 Definition. Let $S$ be a topological semigroup with an identity element 1 and such that, for every neighbourhood $U$ of 1 , we have

(a) $x \in \operatorname{int}\left[U^{-1}(U x) \cap(x U) U^{-1}\right]$ for all $x$ in $S$,

(b) $1 \in \operatorname{int}\left(U^{-1} v \cap w U^{-1}\right)$ for some $w$ and $v$ in $U$.

Following [13], if $S$ is also locally compact, we say $S$ is a stip.

The class of semigroups called stips is very large-see for example [5] and [15]. Their importance partly arises from the fact that every topological semigroup, $S$, with an identity element and such that $S$ coincides with the foundation of $M_{a}(S)$, satisfies items (a) and (b) of Definition 2.4--see for example [5, Chapter 3, Theorem 1.2].

2.5 Some aspects of stips. For ease of reference we briefly list some results on stips. Full details on these can be found in [13] and [5]. Let $S$ be a stip with an identity element 1 , throughout this section.

(i) If $W$ is a compact neighbourhood of 1 , we can find a sequence of open neighbourhoods of $1,\left\{W_{k}\right\}$, and a pseudometric, $\rho$, such that

$$
\begin{aligned}
& W_{k+1}^{-1}\left(W_{k+1} x\right) \subseteq\left\{y \in S: \rho(y, x) \leqslant 2^{-k+1} \subseteq W_{k}^{-1}\left(W_{k} x\right),\right. \\
& \text { and } \rho(x z, y z) \leqslant \rho(x, y)
\end{aligned}
$$

for all $x, y, z \in S$ and $k \in N$.

(ii) One can employ a standard argument to show that, for a function $f$ in $C(S)$ we have that

$f \in L U C(S)$ if and only if, given $\varepsilon>0$ we can find a neighbourhood 0 of 1 such that $0 x \cap 0 y \neq \emptyset$ implies $|f(x)-f(y)|<\varepsilon \quad(x, y \in S)$.

(iii) Let $E$ be the set of all idempotent elements of $S$. For every $e$ in $E$, we have that $e S e$ is a stip with identity element $e$. We say $e$ is a $\delta$-isolated idempotent if $\{e\}$ is a $G_{\delta}$-set in $e S e \cap E$.

2.6 Definitions. Let $S$ be a $C$-distinguished topological semigroup.

$$
\begin{aligned}
P(S) & :=\{\mu \in M(S):\|\mu\|=\mu(S)=1\} \\
P_{c}(S) & :=\{\nu \in P(S): \operatorname{supp}(\nu) \text { is compact }\}
\end{aligned}
$$

and let $A$ be a subspace of $M(S)^{\star}$ such that $\mu o h \in A$ for all $\mu$ in $M(S)$ and $h$ in $A$.

We say $m \in A^{\star}$ is a mean on $A$ if

$$
\|m\|=m(e)=1 \text { and } m(f) \geqslant 0 \text { for every non-negative } f \text { in } A \text {. }
$$

A left invariant mean $(L I M)$ on $A$ is a mean on $A$ such that

$$
m\left(\delta_{x} o h\right)=m(h) \text { for all } x \in S \text { and } h \in A \text {. }
$$


A topologically left invariant mean $(T L I M)$ on $A$ is a mean $m$ on $A$ such that

$$
m(\mu o h)=m(h) \quad(\mu \in P(S) \text { and } h \in A) .
$$

We denote the set of all $L I M \mathrm{~s}$ (respectively, TLIM s on $A$ by $L I M(A)$ (respectively $\operatorname{TLIM}(A))$.

\section{Statement of Results}

3.1 TheOREM. Let $S$ be a non-relatively neo-compact stip with $L U C(S)$ admitting a left invariant mean. Then

$$
\operatorname{card}(L I M(L U C(S))) \geqslant 2^{2^{d(S)}} .
$$

For certain stips we improve the preceding result as follows:

3.2 ThEOREM. Let $S$ be a non-relatively neo-compact stip with $L U C(S)$ admitting a left invariant mean. Then

(i) $2^{2^{b(S)}} \geqslant \operatorname{card}(\operatorname{LIM}(L U C(S))) \geqslant 2^{2^{d(S)}}$ if $S$ is either commutative or has a $\delta$-isolated identity;

(ii) $\operatorname{card}(L I M(L U C(S)))=2^{2^{b(S)}}$ if $S$ is either $\sigma$-compact or as in (i) but with $C^{-1} D$ and $D C^{-1}$ compact, for all compact subsets $C$ and $D$ of $S$.

For semigroups admitting absolutely continuous measures, we have the following Theorem for more general topological senigroups-including cases where the senigroup may not be locally compact.

3.3 Theorem. Let $S$ be a non-relatively neo-compact $C$-distinguished topological semigroup such that $M_{a}(S)$ is non-zero. Then

(i) if $L U C(S)$ admits a left invariant mean we have that

$$
2^{2^{b(S)}} \geqslant \operatorname{card}(L I M(L U C(S))) \geqslant 2^{2^{d(S)}}
$$

(ii) if $M(S)^{\star}$ admits a left topologically invariant mean, we have that

$$
2^{2^{b(S)}} \geqslant \operatorname{card}\left(T L I M\left(M(S)^{\star}\right)\right) \geqslant 2^{2^{d(S)}} .
$$

In particular the inequalities in both (i) and (ii) reduce to equalities if, in addition, either

(a) $S$ is $\sigma$-compact;

or (b) $S$ is such that $C^{-1} D$ and $D C^{-1}$ are compact, whenever $C$ and $D$ are compact subsets of $S$. 
3.4 COROLLARY. Let $S$ be a non-compact topological semigroup with an identity element such that $S$ coincides with the foundation of $M_{a}(S)$, the sets $C^{-1} D$ and $D C^{-1}$ are compact whenever $C$ and $D$ are compact subsets of $S$. If $L U C(S)$ admits a left invariant mean, then

$$
\operatorname{card}\left(T L I M\left(M\left(S^{\star}\right)\right)\right)=\operatorname{card}\left(T L I M\left(M_{a}(S)^{\star}\right)\right)=\operatorname{card}(L I M(L U C(S)))=2^{2^{d(S)}}
$$

In particular we have the following corollary which also contains the result of Lau and Paterson [10].

3.5 Corollary. Let $G$ be a non-compact locally compact group such that $L U C(G)$ admits a left invariant mean. Then

$$
\operatorname{card}\left(T L I M\left(M(G)^{\star}\right)\right)=\operatorname{card}\left(T L I M\left(L^{\infty}(G)\right)\right)=\operatorname{card}(L I M(L U C(G)))=2^{2^{d(G)}}
$$

The next result is of particular interest for situations where the underlying topological semigroup $S$ does not support absolutely continuous measures and does not have the "nice" topological structure of a stip.

3.6 Theorem. Let $S$ be a $C$-distinguished topological semigroup that is $\sigma$ compact and not relatively neo-compact. Then, if $M(S)^{\star}$ admits a topologically left invariant mean, we have that

$$
\operatorname{card}\left(T L I M\left(M(S)^{\star}\right)\right) \geqslant 2^{c}
$$

where $c$ denotes the cardinality of the continuum.

\section{SOME KEY LEMMAS}

A proof of the following lemma can be found in [3] for the case of a locally compact semigroup. However, one can easily note that the proof also holds for our case.

4.1 Lemma. Let $T$ be a Borel subset of a $C$-distinguished topological semigroup, $S$, and suppose that $M(S)^{\star}$ admits a $T L I M$. Then the following items are equivalent:

(i) there exists a $T L I M, m$, on $M(S)^{\star}$ such that $m\left(\chi_{T}\right)=1$;

(ii) for each $\nu$ in $P(S)$ and $\varepsilon>0$, we can find a point $x$ in $S$ such that $\nu^{\star} \delta_{x}(T)>1-\varepsilon$.

A mild adjustment (which we omit) of the argument given in [3] yields the following analogue of Lemma 4.1 for $L I M$ on $L U C(S)$. 
4.2 LEMMA. Let $T$ be a subset of a $C$-distinguished topological semigroup $S$ and suppose that $L U C(S)$ admits a $L I M$. Then the following are equivalent:

(i) there exists a $T L I M, m$, on $L U C(S)$ such that $m(f)=1$, for every $f \in L U C(S)$ with $0 \leqslant f \leqslant 1$ and $f=1$ on $T$;

(ii) for each $\nu$ in $P_{\mathrm{c}}(S)$ and $\varepsilon>0$, we can find $x$ in $S$ such that $\nu^{\star} \delta_{x}(f)>$ $1-\varepsilon$, for every $f \in L U C(S)$ with $0 \leqslant f \leqslant 1$ and $f=1$ on $T$.

Our next two lemmas together generalise [10, Lemma 1 and Proposition 1].

4.3 LEMMA. Let $S$ be a non-relatively neo-compact $C$-distinguished topological semigroup and $V$ be a compact subset. Let $\alpha$ be the smallest ordinal having cardinality $d(S)$ and $\left\{C_{\beta}: 1 \leqslant \beta<\alpha\right\}$ be a family of relatively neo-compact neighbourhoods covering $S$ and closed under finite unions. Then

(i) there exists a subset $\left\{x_{\beta \gamma}: 1 \leqslant \beta \leqslant \gamma<\alpha\right\}$ of $S$ such that the sets $\left\{V^{-1}\left(V C_{\gamma} x_{\beta \gamma}\right): 1 \leqslant \beta \leqslant \gamma<\alpha\right\}$ are pairwise disjoint;

(ii) if $T_{\beta}:=\cup\left\{C_{\gamma} x_{\beta \gamma}: \beta \leqslant \gamma<\alpha\right\}$, we have that $\bar{T}_{\beta}$ satisfies item (ii) of both Lemma 4.1 and 4.2 .

ProOF: (i) (see [10]). We employ a transfinite inductive argument based on the well-ordered set $\{(\gamma, \beta): 1 \leqslant \beta \leqslant \gamma<\alpha\}$ where

$$
\left(\gamma_{1}, \beta_{1}\right)<\left(\gamma_{2}, \beta_{2}\right) \text { means } \gamma_{1}<\gamma_{2} \text { or } \gamma_{1}=\gamma_{2} \text { and } \beta_{1}<\beta_{2} \text {. }
$$

Let $x_{11}$ be an arbitarily chosen element of $S$. By the inductive hypothesis, suppose that $x_{\beta \gamma}$ has been chosen for all pairs $(\gamma, \beta)<\left(\gamma_{0}, \beta_{0}\right)$ with $1 \leqslant \beta \leqslant \gamma$. Setting $H:=\bigcup\left\{V C_{\gamma} x_{\beta \gamma}:(\gamma, \beta)<\left(\gamma_{0}, \beta_{0}\right)\right\}$ we have that $H:=\left(V C_{\gamma_{0}}\right)^{-1}(V H)$ admits a covering, by relatively neo-compact sets, of cardinality less than $d(S)$. So we can choose $x_{\beta_{0} \gamma_{0}}$ in $S \backslash H$, which gives our inductive step and the result follows

(ii) Let $\nu$ be any measure in $P_{c}(S)$ and choose $\gamma$ with $\beta \leqslant \gamma<\alpha$ such that $\operatorname{supp}(\nu) \subset C_{\gamma}$. Then for $x=x_{\beta \gamma}$ we have

$$
\operatorname{supp}\left(\nu^{\star} \delta_{x}\right) \subset \operatorname{closure}\left(C_{\gamma} x\right) \subset \bar{T}_{\beta} \text { and so } \nu^{\star} \delta_{x}\left(\bar{T}_{\beta}\right)=1
$$

This completes our proof.

4.4 Lemma. Assume the notation of Lemma 4.3 and let $\Gamma:=$ $\{\beta: \beta$ is an ordinal with $1 \leqslant \beta<\alpha\}$. Then if $S$ is either

(a) a stip

or (b) such that $M_{a}(S)$ is non-zero, 
the subset $V$ can be chosen so that for every $\Gamma_{1} \subseteq \Gamma$, defining $T_{\left(\Gamma_{1}\right)}:=\cup\left\{T_{\beta}: \beta \in \Gamma_{1}\right\}$ we get:

(i) $\bar{T}_{\left(\Gamma_{1}\right)}=\cup\left\{\bar{T}_{\beta}: \beta \in \Gamma_{1}\right\}$;

(ii) there exists a function $f_{\Gamma_{1}}$ in $L U C(S)$ such that $0 \leqslant f_{\Gamma_{1}} \leqslant 1, f_{\Gamma_{1}}=1$ on $\bar{T}_{\left(\Gamma_{1}\right)}$ and for $\Gamma_{2} \subseteq \Gamma$ with $\Gamma_{1} \cap \Gamma_{2}=\emptyset$, we have $f_{\Gamma_{1}} \bullet f_{\Gamma_{2}}=0$.

Proof: Let $\Gamma_{1} \subseteq \Gamma$ be fixed.

(a) We now prove (i) and (ii) for the case where $S$ is a stip. First, by [5, Chapter 3, Theorem 2.6] or [13], we can choose sets $V$ and $W$ as follows:

$$
\left\{\begin{array}{l}
\text { Let } Z \text { and } W \text { be compact neighbourhoods of the identity } \\
\text { of } S \text { and } z \in Z \text { such that } \\
\quad W \subseteq Z^{-1} z \cap Z \\
\text { We fix } V:=Z^{2} \text { and } W \text { to be the set stated in } 2.5(\mathrm{i})
\end{array}\right.
$$

To prove (i), let $x$ be an element of $\bar{T}_{\left(\Gamma_{1}\right)}$. By definition of a stip, $V^{-1}(V x)$ is a neighbourhood of $x$ and so, there exists $\eta \in \Gamma_{1}$ with $V^{-1}(V x) \cap T_{\eta} \neq \emptyset$. So for some $\gamma$ with $\eta \leqslant \gamma<\alpha$, we have $V^{-1}(V x) \cap C_{\gamma} x_{\eta_{\gamma}} \neq \emptyset$. Consequently

$$
x \in V^{-1}\left(V C_{\gamma} x_{\eta_{\gamma}}\right) \text {. }
$$

From (1) and Lemma 4.3(i), we must have

$$
V^{-1}(V x) \cap C_{\tau} x_{\beta \tau}=\emptyset \text { for } \beta \neq \eta
$$

and so

$$
V^{-1}(V x) \cap T_{\beta}=\emptyset \text { for } \beta \neq \eta
$$

Thus, if $0(x)$ denotes the interior of $V^{-1}(V x)$, then

$$
\bar{T}_{(\Gamma \backslash\{\eta\})} \subset S \backslash 0(x)
$$

and so we must have

$$
x \in \bar{T}_{\eta}
$$

Hence $\overleftarrow{T}_{\left(\Gamma_{1}\right)} \subset \cup\left\{\bar{T}_{\beta}: \beta \in \Gamma_{1}\right\}$. The reverse inclusion follows trivially. This proves (i).

Next we prove (ii). Consider the function $f_{\Gamma_{1}}$ defined by

$$
f_{\Gamma_{1}}(x):=1-\min \left(\rho\left(x, \bar{T}_{\left(\Gamma_{1}\right)}\right), 1\right) \quad(x \in S)
$$

where $\rho$ is the pseudometric introduced in item 2.5(i). Trivially, we have that $f_{\Gamma_{1}}$ is continuous, $0 \leqslant f_{\Gamma_{1}} \leqslant 1$ and $f_{\Gamma_{1}}=1$ on $\bar{T}_{\left(\Gamma_{1}\right)}$. 
To show that $f_{\Gamma_{1}}$ is $L U C(S)$, let $\varepsilon>0$ be given. By 2.5(i) and [13] (or [5, Chapter 3]) we can choose open neighbourhoods of the identity, 0 and $U$, such that

$$
\left\{\begin{array}{l}
0 \subseteq U \subseteq W \\
0^{-1}(0 x) \subseteq u^{-1}(U x) \text { for some } u \in U \\
\text { and } 0 y \cap 0 x \neq \emptyset \text { implies } \rho(x, y)<\varepsilon(x, y \in S)
\end{array}\right.
$$

Now suppose $0 y \cap 0 x \neq \emptyset$, and consider the case where $f_{\Gamma_{1}}(x) \neq \emptyset$. Then $\rho\left(x, \bar{T}_{\left(\Gamma_{1}\right)}\right)<$ 1 and so $\rho\left(x, T_{\beta}\right)<1$ for some $\beta \in \Gamma_{1}$. So we can find a $\gamma$ with $\beta \leqslant \gamma<\alpha$ and $\rho\left(x, C_{\gamma} x_{\beta \gamma}\right)<1$. By $2.5(\mathrm{i})$ and $\left({ }^{*}\right)$, we get

$$
x \in W^{-1}\left(W C_{\gamma} x_{\beta \gamma}\right) \subset z^{-1}\left(Z W C_{\gamma} x_{\beta \gamma}\right) \subset z^{-1}\left(V C_{\gamma} x_{\beta \gamma}\right)
$$

Since $0 x \cap 0 y \neq \emptyset$, we have (by (2) and $(*)$ ) that

$$
y \in 0^{-1}(0 x) \subset u^{-1}(U x) \subset u^{-1}\left(\left(Z^{-1} z\right) x\right) \subset(Z u)^{-1}(z x)
$$

So there exists $z_{1} \in Z$ such that

$$
z_{1} u y=z x
$$

From (3) and (4) we get $z_{1} u y \in V C_{\gamma} x_{\beta \gamma}$ and, since $z_{1} u \in Z U \subset Z^{2}=V$, we thus have

$$
y \in V^{-1}\left(V C_{\gamma} x_{\beta \gamma}\right)
$$

Hence (3) and (5) say that both $x$ and $y$ are in $V^{-1}\left(V C_{\gamma} x_{\beta \gamma}\right)$. Consequently, recalling Lemma 4.3 , if $\rho\left(y, C_{\gamma} x_{\beta \gamma}\right)<1$ we get

$$
\left|f_{\Gamma_{1}}(x)-f_{\Gamma_{1}}(y)\right|=\left|\rho\left(x, C_{\gamma} x_{\beta \gamma}\right)-\rho\left(y, C_{\gamma} x_{\beta \gamma}\right)\right| \leqslant \rho(x, y)<\varepsilon ;
$$

and if $\rho\left(y, C_{\gamma} x_{\beta_{\gamma}}\right) \geqslant 1$, we get $f_{\Gamma_{1}}(y)=0$ and

$$
\left|f_{\Gamma_{1}}(x)-f_{\Gamma_{1}}(y)\right|=\left|1-\rho\left(x, C_{\gamma} x_{\beta \gamma}\right)\right| \leqslant\left|\rho\left(y, C_{\gamma} x_{\beta \gamma}\right)-\rho\left(x, C_{\gamma} x_{\beta \gamma}\right)\right| \leqslant \rho(x, y)<\varepsilon .
$$

Therefore $0 x \cap 0 y \neq \emptyset$ and $f_{\Gamma_{1}}(x) \neq 0$ imply that $\left|f_{\Gamma_{1}}(x)-f_{\Gamma_{1}}(y)\right|<\varepsilon$.

Now suppose $0 x \cap 0 y \neq \emptyset$ and $f_{\Gamma_{1}}(x)=0$. If $f_{\Gamma_{1}}(y)=0$, there is nothing to prove, while if $f_{\Gamma_{1}}(y) \neq 0$ then (as noted above) we can find $\eta \in \Gamma_{1}$, and suitable $\tau$ such that $\rho\left(y, C_{\tau} x_{\eta_{\tau}}\right)<1$; leading to

$$
\left|f_{\Gamma_{1}}(x)-f_{\Gamma_{1}}(y)\right|=\left|1-\rho\left(y, C_{\tau} x_{\eta_{\tau}}\right)\right| \leqslant \rho(x, y)<\varepsilon .
$$


Hence $0 x \cap 0 y \neq \emptyset$ implies $\left|f_{\Gamma_{1}}(x)-f_{\Gamma_{1}}(y)\right| \leqslant \varepsilon(x, y \in S)$; and so $f_{\Gamma_{1}} \in L U C(S)$, by item 2.5(ii).

Next we consider the function $f_{\Gamma_{1}} \bullet f_{\Gamma_{2}}$ where $\Gamma_{1}$ and $\Gamma_{2}$ are given disjoint subsets of $\Gamma$. Suppose

$$
\left(f_{\Gamma_{1}} \cdot f_{\Gamma_{2}}\right)(x)=f_{\Gamma_{1}}(x) f_{\Gamma_{2}}(x) \neq 0 \text { for some } x \in S
$$

Then one can find $\beta \in \Gamma_{1}$ and $\eta \in \Gamma_{2}$ such that

$$
\rho\left(x, T_{\beta}\right)<1 \text { and } \rho\left(x, T_{\eta}\right)<1
$$

This would lead to

$$
x \in V^{-1}\left(V C_{\gamma} x_{\beta \gamma}\right) \cap V^{-1}\left(V C_{\tau} x_{\eta_{\tau}}\right)
$$

for some $\gamma$ and $\tau$ with $\beta \leqslant \gamma<\alpha$ and $\eta \leqslant \tau<\alpha$. But (6) contradicts Lemma 4.3. By this contradiction we conclude that the function $f_{\Gamma_{1}} \bullet f_{\Gamma_{2}}$ is identically equal to the zero function.

This completes our proof when $S$ is a stip.

(b) Now we consider the case where $M_{a}(S)$ is non-zero. By [5, Chapter 2, Theorems 1.14 and 2.3] or [4], we can find a measure $\nu$ in $P_{c}(S) \cap M_{a}(S)$ such that the mapping $x \rightarrow \nu^{\star} \delta_{x}$ of $S$ into $M_{a}(S)$ is norm continuous. Let $D:=\operatorname{supp}(\nu)$ and note that $D$ is compact.

We define $V:=D^{2}$. By [4] or [5, Chapter 2, Lemma 1.9], we have $V^{-1}(V x)$ a neighbourhood of $x$, for all $x \in S$. Hence, with our newly defined $V$, item (i) can be proved as done in case (a) and so we omit the details.

To prove (ii), we define the function $f_{\Gamma_{1}}$ by

$$
f_{\Gamma_{1}}(x):=\nu^{\star} \delta_{x}\left(\overline{D T}_{\left(\Gamma_{1}\right)}\right) \quad(x \in S)
$$

For any $x, y \in S$ we have that

$$
\left\|_{x}\left(f_{\Gamma_{1}}\right)-{ }_{y}\left(f_{\Gamma_{1}}\right)\right\| \leqslant\left\|\nu^{\star} \delta_{x}-\nu^{\star} \delta_{y}\right\|
$$

and so, by the norm continuity of the map $s \rightarrow \nu^{\star} \delta_{s}$ of $S$ into $M(S)$, we have $f_{\Gamma_{1}}$ in $L U C(S)$. It is also evident that $0 \leqslant f_{\Gamma_{1}} \leqslant 1$ and $f_{\Gamma_{1}}=1$ on $\bar{T}_{\left(\Gamma_{1}\right)}$.

To complete the proof we now show that $f_{\Gamma_{1}} \bullet f_{\Gamma_{2}}=0$ where $\Gamma_{2} \subseteq \Gamma$ is disjoint from $\Gamma_{1}$. To this end, suppose on the contrary there exists an $x$ in $S$ such that

$$
f_{\Gamma_{1}}(x) f_{\Gamma_{2}}(x)>0
$$


That $f_{\Gamma_{1}}(x)>0$ means

$$
\nu^{\star} \delta_{x}\left(\overline{D T}_{\left(\Gamma_{1}\right)}\right)=\nu^{\star} \delta_{x}\left(D x \cap \overline{D T}_{\left(\Gamma_{1}\right)}\right)>0
$$

since $\operatorname{supp}\left(\nu^{\star} \delta_{x}\right)=D x$. By [4] or [5] (as similarly noted above) we have that $D^{-1}\left(D^{2} x\right)$ is a neighbourhood of $D x$ and so $D^{-1}\left(D^{2} x\right) \cap D T_{\left(\Gamma_{1}\right)} \neq \emptyset$ which implies that

$$
x \in D^{-2}\left(D^{2} T_{\left(\Gamma_{1}\right)}\right)=V^{-1}\left(V T_{\left(\Gamma_{1}\right)}\right)
$$

Similarly, that $f_{\Gamma_{2}}(x)>0$ implies

$$
x \in V^{-1}\left(V T_{\left(\Gamma_{2}\right)}\right)
$$

By Lemma 4.3, we have

$V^{-1}\left(V T_{\left(\Gamma_{1}\right)}\right) \cap V^{-1}\left(V T_{\left(\Gamma_{2}\right)}\right)=\emptyset$-contrary to $(7)$ and (8). By this contradiction, we conclude that $f_{\Gamma_{1}} \bullet f_{\Gamma_{2}}$ is the zero function on $S$.

\section{Proof of Theorems 3.1 and 3.2}

Throughout this section, $S$ denotes a stip that is not relatively neo-compact.

5.1 Proof of Theorem 3.1: We employ an argument along lines given in [9]. Let $\Gamma$ be as stated in Lemma 4.4 and let $U$ be the class of ultrafilters on $\Gamma$.

Corresponding to each $A \in U$ we claim that there exists a $L I M$ on $L U C(S)$, $m_{A}$, such that

$$
m_{A}\left(f_{\Gamma_{1}}\right)=1 \text { for every } \Gamma_{1} \in A
$$

where $f_{\Gamma_{1}}$ is as in Lemma 4.4. To verify our claim, we first note that for each $\beta \in \Gamma$ we can find $m_{\beta} \in L I M(L U C(S))$ such that

$$
m_{\beta}\left(f_{\{\beta\}}\right)=1 \text {, by Lemmas } 4.2 \text { and } 4.4 \text {. }
$$

So for each $\Gamma_{1} \in A$ we can choose $m_{\Gamma_{1}} \in L I M\left(L U C(S)\right.$ ) (for example $m_{\Gamma_{1}}=m_{\beta}$ for any $\beta \in \Gamma_{1}$ ) such that

$$
m_{\Gamma_{1}}\left(f_{\Gamma_{1}}\right)=1
$$

Since $L I M(L U C(S))$ is a weak ${ }^{\star}$-compact subset of $L U C(S)^{\star}$, there exists a weak * limit point, $m \in L I M(L U C(S))$, of the net $\left(m_{\Gamma_{1}}\right)_{\Gamma_{1 \in A}}$, where the ordering on $A$ is backwards inclusion. One can easily note that $m$ satisfies (1) and so we take $m_{A}:=m$ and our ciaim is proved. 
Next we show that, for distinct ultrafilters $A_{1}$ and $A_{2}$ in $U$, we have $m_{A_{1}} \neq m_{A_{2}}$. To this end, fix disjoint subsets $\Gamma_{1}$ and $\Gamma_{2}$ of $\Gamma$ with $\Gamma_{1} \in A_{1}$ and $\Gamma_{2} \in A_{2}$. Let $f_{\Gamma_{1}}$ and $f_{\Gamma_{2}}$ be as in Lemma 4.4 and note that $0 \leqslant f_{\Gamma_{1}}+f_{\Gamma_{2}} \leqslant 1$. So item (1) and Lemma 4.4 (ii) give

$$
1 \geqslant m_{A_{1}}\left(f_{\Gamma_{1}}+f_{\Gamma_{2}}\right)=m_{A_{1}}\left(f_{\Gamma_{1}}\right)+m_{A_{1}}\left(f_{\Gamma_{2}}\right)=1+m_{A_{1}}\left(f_{\Gamma_{2}}\right)
$$

and so $m_{A_{1}}\left(f_{\Gamma_{2}}\right)=0$, while $m_{A_{2}}\left(f_{\Gamma_{2}}\right)=1$.

It follows that $\operatorname{card}(L I M(L U C(S))) \geqslant \operatorname{card}(U)$. It is well-known that $\operatorname{card}(U) \geqslant$ $2^{2^{|\Gamma|}}$ (see for example $[7]$ ). As $|\Gamma|=d(S)$, we have

$$
\operatorname{card}(L I M(L U C(S))) \geqslant 2^{2^{d(S)}}
$$

5.2 Proof of Theorem 3.2: (i) By Theorem 3.1 we only need to show that

$$
\operatorname{card}(L I M(L U C(S))) \leqslant 2^{2^{b(S)}}
$$

First we consider the case where the identity or $S$ is $\delta$-isolated. By $[14]$ or [15, Chapter 3, Proposition 3.8(i)] we can find a compact subgroup $G$ of $S$ such that every compact subset $C$ of $S$ contains a countable subset $B_{C}$ such that

$$
C \subseteq \overline{G B}_{C}
$$

Let $\pi$ be normalised Haar measure on $G$ and define the space

$$
N(S):=\{\pi \circ f: f \in L U C(S)\}
$$

For $g \in G$ and $b \in B_{C}$, we have (by invariance of $\pi$ )

$$
\pi \circ f(g b)=\pi \circ f(b)
$$

So each function in $N(S)$ is uniquely determined by its value on

$$
B:=\cup\left\{B_{C_{\gamma}}: 1 \leqslant \gamma<\alpha^{1}\right\}
$$

where $\alpha^{1}$ is the smallest ordinal having cardinality $b(S)$ and $\left\{C_{\gamma}: 1 \leqslant \gamma<\alpha^{1}\right\}$ is a covering of $S$ by compact sets. Hence

$$
|B| \leqslant \aleph_{0} \bullet b(S)=b(S)
$$


Therefore

$$
|N(S)| \leqslant c^{|B|} \leqslant c^{b(S)}=2^{b(S)}
$$

and

$$
\left|N(S)^{\star}\right| \leqslant 2^{2^{b(S)}}
$$

Now for each $m \in \operatorname{LIM}(\operatorname{LUC}(S))$, we have that $m$ is a $T L I M$ by $[\mathbf{5}$, Chapter 4 , Lemma 2.3], and so

$$
m(f)=m(\pi \circ f) \quad(f \in L U C(S)) .
$$

Thus $m$ can be viewed as an element of $N(S)^{\star}$ and, recalling (1), we get

$$
\operatorname{card}(L I M(L U C(S))) \leqslant \operatorname{card}\left(N(S)^{\star}\right) \leqslant 2^{2^{b(S)}} .
$$

For the case where $S$ is commutative, let $e$ be a $\delta$-isolated idempotent and note that $m \in L I M(L U C(S))$ gives $m \in L I M(L U C(e S e))$ since ${ }_{e} f_{e}={ }_{e} f$ and $m(e f)=$ $m(f)$. Since $e S e$ is a stip (see [5] or [13]) with $\delta$-isolated identity $e$, our result follows from the above argument.

(ii) If $S$ is $\sigma$-compact, by [13] or [5, Chapter 3, Lemma 3.4] we can find $x \in S$ and a $\delta$-isolated idempotent, $e$, such that

$$
x S \subset e S e
$$

and so each $m \in \operatorname{LIM}(L U C(S))$ gives rise to $m \in L I M(L U C(e S e))$, leading to

$$
\operatorname{card}(L I M(L U C(S))) \leqslant 2^{2^{b(S)}}
$$

as noted above.

Recalling (i) and Proposition 2.2, our result follows.

6 Proof of Theorem 3.3 and Corollaries 3.4 and 3.5

6.1 Proof of Theorem 3.3: (i) The proof of Theorem 3.1 gives

$$
\operatorname{card}(L I M(L U C(S))) \geqslant 2^{2^{\mathrm{d}(S)}}
$$

for our present senugroup $S$, and so we omit details.

To show that $\operatorname{card}(\operatorname{LIM}(L U C(S))) \leqslant 2^{2^{b(S)}}$ we first develop a background that would enable one to argue in a similar manner to the proof of Theorem 3.2(i). By [4] or [5], we can find a measure $\nu$ in $P_{c}(S) \cap M_{a}(S)$ such that the mapping $x \rightarrow \nu^{\star} \delta_{x}$ of 
$S$ into $M(S)$ is norm continuous. Hence each compact subset $C$ contains a countable subset $B_{C}$ such that

$$
\left\{\nu^{\star} \delta_{x}: x \in C\right\} \subseteq \text { closure }\left\{\nu^{\star} \delta_{x}: x \in B_{C}\right\}
$$

Consequently, noting that $\nu o f(x)=\nu^{\star} \delta_{x}(f)$, we have for all $f \in L U C(S)$,

$$
\{\nu o f(x): x \in C\} \subset \text { closure }\left\{\nu o f(x): x \in B_{C}\right\},
$$

Let

$$
N(S):=\{\nu o f: f \in L U C(S)\}
$$

and note that $m(\nu \circ f)=m(f)$ for all $f \in L U C(S)$ and $m \in L I M(L U C(S))$. With this and (1) in mind, the reader can mimic the argument in the proof of Theorem 3.2(i) to complete the proof.

(ii) Note that for $h \in M(S)^{\star}$ and $\nu$ as in the proof of (i), $\nu o h$, viewed as a function on $S$ (given by $\nu o h(x)=\nu o h\left(\delta_{x}\right)$ ) is in $L U C(S)$ since

$$
\|\|_{x}(\nu o h)-{ }_{y}(\nu o h)\|\leqslant\| h\|\| \nu^{\star} \delta_{x}-\nu^{\star} \delta_{y} \| \quad(x, y \in S) .
$$

So each $T L I M$ on $M(S)^{\star}$ can be viewed as a $L I M$ on $L U C(S)$ and hence

$$
\operatorname{card}\left(T L I M\left(M(S)^{\star}\right)\right) \leqslant 2^{2^{b(S)}}
$$

by (i). Also the proof of Theorem 3.1 can be mildly amended to give

$$
\operatorname{card}\left(T L I M\left(M(S)^{\star}\right)\right) \geqslant 2^{2^{d(S)}},
$$

by using Lemma 4.1 in place of 4.2 and replacing the functions $f_{\Gamma_{1}} \in L U C(S)$ by functionals $f_{\Gamma_{1}} \in M(S)^{\star}$ given by $f_{\Gamma_{1}}(\mu):=\mu\left(\bar{T}_{\left(\Gamma_{1}\right)}\right)$ for all $\mu \in M(S)$. (We omit the details.)

Recalling Proposition 2.2, the proof of our Theorem is complete.

6.2 Proof of Corollaky 3.4: By restriction each TLIM on $M(S)^{\star}$ gives rise to a $T L I M$ on $M_{a}(S)^{\star}$ and on $L U C(S)$. By [5, Chapter 4, Theorem 2.4] each $L I M$ on $L U C(S)$ gives rise to a $T L I M$ on $M(S)^{\star}$. With this in mind, the result follows from Theorem 3.3 .

6.3 Proof of Corollary 3.5: Recalling that $M_{a}(G)$ is identifiable with $L(G)$ and hence $M_{a}(G)^{\star}$ with $L^{\infty}(G)$-see for example [8], the result is a special case of Corollary 3.4. 


\section{Proof of Theorem 3.6}

Since $S$ is $\sigma$-compact, we have $b(S)=d(S)=\aleph_{0}$ and the sets $C_{\gamma}$ mentioned in Lemma 4.3 can thus be chosen to be compact, giving $\sigma$-compact sets $T_{\beta}$ for $1 \leqslant \beta<\infty$. Hence mimicing the proof of Theorem 3.1, using Lemma 4.1 in place of 4.2 and replacing functions $f_{\Gamma_{1}}$ by functionals given by $f_{\Gamma_{1}}(\mu):=\mu\left(T_{\left(\Gamma_{1}\right)}\right)$ for all $\mu \in M(S)$ and $\Gamma_{1} \subset \Gamma$, we get

$$
\operatorname{card}\left(T L I M\left(M(S)^{\star}\right)\right) \geqslant 2^{2^{d(S)}}=2^{2^{\aleph_{0}}}=2^{c}
$$

(We omit details.)

\section{SOME REMARKS}

8.1. As noted in 2.4 , interest in stips arose from the fact that every locally compact topological semigroup, $S$, with an identity element and coinciding with the foundation of $M_{a}(S)$, is a stip. However, it is still not known whether every stip supports an absolutely continuous measure-see [5] and [13]. Recently a number of studies have shown that many results one can obtain for locally compact topological semigroups supporting absolutely continuous measures remain valid for stips-see for example [5, Chapter 3, Section 4] and [14]. To some extent out Theorems 3.1 and 3.2 belong to the same sphere.

8.2. We strongly believe that Theorem 3.2(i) holds for all stips. A reader interested in persuing this problem may find the studies in [5] and [13] of some use.

8.3. There are many semigroups satisfying the hypothesis of Theorem 3.6 which are not stips and do not support absolutely continuous measures. One simple example is $S:=[0, \infty)$ with the relative line topology and maximum operation. We also feel that Theorem 3.6 may be extendable to left invariant means on left uniformly continuous functions as follows:

CONJECTURE. If $S$ is a $C$-distinguished topological semigroup that is $\sigma$-compact but not relatively neo-compact, and such that $L U C(S)$ admits a left invariant mean, then

$$
\operatorname{card}(L I M(L U C(S))) \geqslant 2^{c}
$$

\section{REFERENCES}

[1] C. Chou, 'On topologically invariant means on a locally coinpact group', Trans. Amer. Math. Soc. 151 (1970), 443-456.

[2] C. Chou, 'The exact cardinality of the set of invariant means on a group', Proc. Amer. Math. Soc. 55 (1976), 103-106.

[3] M.M. Day, 'Lumpy subsets in left-amenable locally compact semigroups', Pacific J. Math. 62 (1976), 87-92. 
[4] H.A.M. Dzinotyiweyi, 'Algebras of measures on $C$-distinguished topological semigroups', $M a t h$. Proc. Cambridge Philos. Soc. 84 (1978), 323-336.

[5] H.A.M. Dzinotyiweyi, The analogue of the group algebra for topological semigroups (Pitman Books Ltd., Boston, London, Melbourne, 1984).

[6] H.A.M. Dzinotyiweyi, 'Nonseparability of quotient spaces of function algebras on topological semigroups', Trans. Amer. Math. Soc. 272, 223-235.

[7] L. Gillman and M. Jerison, Rings of continuous functions (Van Nostrand, New York, 1960).

[8] E. Hewitt and K.A. Ross, Abstract harmonic analysis Vol. 1 (Springer-Verlag, Berlin, 1963 ).

[8] M.M. Klawe, 'Dimensions of the sets of invariant means of semigroups', Illinois J. Math. 24 (1980), 233-243.

[10] A.T.M. Lau and A.L.T. Paterson, 'The exact cardinality of the set of topological left invariant means on an amenable locally compact group', Proc. Amer. Math. Soc. 98 (1986), 75-80.

[11] T.S. Liu and A. Van Rooij, 'Invariant means on a locally compact group', Monatsh. Math. 78 (1974), 356-359.

[12] J.M Rosenblatt, 'The number of extensions of an invariant mean', Composito Math. 33 (1976), 147-159.

[13] G.L.G. Sleijpen, 'Locally compact semigroups and continuous transiations of measures', Proc. London Math. Soc. 37 (1978), 75-97.

[14] G.L.G. Sleijpen, 'A note on measures vanishing on emaciated sets', Bull. London Math. Soc. 13 (1981), 219-223.

University of Zimbabwe

P.O. Box MP 167

Mount Pleasant

Harare

ZIMBABWE 